\title{
Radiation therapy after radical prostatectomy: A single-centre radiation oncology experience in trends of referral and treatment practices
}

\author{
Michel Zimmermann, MD; ${ }^{*}$ Daniel Taussky, MD, ${ }^{* \dagger}$ Guila Delouya, MD, MSc, ${ }^{* \dagger}$ Abdullah M. Alenizi, MD;* \\ Kevin C. Zorn, MD, FRCSC*
}

*Department of Radiation Oncology, Centre hospitalier de I'Université de Montréal (CHUM) - Hôpital Notre-Dame, Montréal, QC; 'Centre de recherche, CHUM, Montréal, QC; *Urology Section, Department of Surgery, CHUM, Montréal, QC

Cite as: Can Urol Assoc J 2015;9(9-10):E608-12. http://dx.doi.org/10.5489/cuaj.2815 Published online September 9, 2015.

\section{Abstract}

Introduction: Our objective was to assess whether referral and treatment practices have changed since publication of the Southwest Oncology Group (SWOG) 8794 Trial in 2009, the first randomized study to demonstrate an overall survival advantage of adjuvant radiation therapy (RT) after radical prostatectomy (RP).

Methods: We retrospectively reviewed all medical charts of men who received RT at our institution between 2004 and 2014 following RP. All RT was conducted by a single radiation oncologist (DT). We divided the cohort into 2 groups according to first referral date before or after the SWOG 8794 trial publication (i.e., before 2010 and after 2010).

Results: Medical charts were available for $161 / 165$ patients $(97.6 \%)$. RP was performed at the same institution in $58 \%$ of cases. The median time between surgery and first referral for RT decreased significantly from 672 days (interquartile range [IQR] 295-1449) before 2010 to 300 days (IQR 225-1023) after 2010 ( $p=0.04)$. This trend was associated with lower median prostate-specific antigen (PSA) at RT referral $(0.26 \mu \mathrm{g} / \mathrm{L}$ [IQR $0.17-0.48]$ vs. $0.46 \mu \mathrm{g} / \mathrm{L}$ [IQR $0.25-0.90]$, respectively; $p=0.001)$. Androgen-deprivation therapy with RT nearly tripled over time from $13 \%$ before 2010 to $37 \%$ after 2010 ( $p=0.003$ ). Throughout the study period, the time interval between surgery and RT initiation was positively correlated with pT-stage $(p=0.001)$, Gleason score $(p=0.005)$ and PSA doubling time $(p<0.001)$.

Conclusions: At our tertiary-referral academic institution, post-RP patients are notably referred earlier for RT and at lower PSA values compared to men treated prior to 2010. Further study is necessary to evaluate this impact on biochemical recurrence-free survival.

\section{Introduction}

Three phase III studies have shown the benefit of adjuvant radiation therapy (RT) over a wait-and-see (salvage) approach after radical prostatectomy (RP) for locally-advanced prostate cancer (T3, Gleason score $\geq 8$ or positive surgical margins). ${ }^{1-3}$ All have demonstrated significant improvement in biochemical recurrence-free survival. Two of these trials reported improvement in progression-free survival. The difference was statistically significant in the Southwest Oncology Group (SWOG) 8794 trial $^{2}$ and borderline significant in the European Organization for Research and Treatment of Cancer trial' (EORTC) 22911 ( $p=0.054)$. With respect to overall survival, only SWOG 8794 achieved significant benefit. ${ }^{2}$ Published in late 2009, with median follow-up of 12.6 years, the SWOG 8794 trial indicated that 9.1 patients needed to be treated to prevent 1 death. Moreover, based on this trial, adjuvant radiotherapy is more cost-effective than observation. ${ }^{4}$

We think that the SWOG 8794 trial might lead urologists and radiation oncologists to progressively change their practice, resulting in patient referrals to RT departments earlier and at lower prostate-specific antigen (PSA) levels. Therefore, we decided to conduct a retrospective study of the practice of a single radiation oncologist over the last 10 years to evaluate our hypothesis at a Canadian tertiaryreferral oncology centre.

\section{Methods}

After institutional review board approval, we retrospectively reviewed all charts of patients who had RT after RP at our institution at the hands of a single radiation oncologist between 2004 and 2014. We tested our hypothesis that surgeons might have progressively changed their practice in the months following publication of the SWOG 8794 trial in 2009. Based on this assumption, we divided our cohort into 2 groups (pre-trial publication and post-trial publication), taking January 1, 2010 as the cut-off date.

We noted patient demographics by chart review, including last PSA count before RP and date of surgery. Pathological $\mathrm{T}$ (pT)-stage (and $\mathrm{N}$ stage when available), combined 
Gleason score and margin status were retrieved from pathology records. The following parameters were recorded: postoperative PSA (nadir), subsequent PSA doubling time (PSADT), date of referral to our radio-oncology department, last PSA count before RT, date of first RT, whether prophylactic radiation to the pelvis and/or concomitant hormone therapy were given.

Postoperative PSADT was calculated from the last 2 or 3 PSA values before RT, irrespective of whether a threshold value $(0.2 \mu \mathrm{g} / \mathrm{L})$ was reached, using the online PSADT calculator developed by the Memorial Sloan Kettering Cancer Center. PSADT subgroups were defined as short ( $<6$ months), intermediate (6-12 months) or long (>12 months).

All patients received external beam radiation of the prostate bed up to a total dose of 66 Gy in 2 Gy/fraction, deploying standard conformal 3D or intensity-modulated radiation therapy with a 4-6 MV linear accelerator. Inclusion of pelvic lymph nodes (and treated up to 44-46 Gy) was left to the discretion of the radiation oncologist.

Follow-up data were extracted from our medical charts. When information was missing, follow-up phone calls to the patients were made to complete the data.

\section{Statistical analysis}

Continuous variables were compared with the MannWhitney test and categorical variables with the Chi-square test (or Fischer's exact test). Pearson and Spearman tests analyzed correlations between 2 variables. Statistical significance was reached at $p \leq 0.05$ in all tests performed. Data were analyzed by SPSS 17.0 for Windows (IBM SPSS, Chicago, IL).

\section{Results}

Between September 2004 and November 2014, 165 consecutive patients were treated after surgery by a single radiation-oncologist (DT) at our tertiary-referral academic institution. In total, 161 charts were available for analysis. Among these, 103 men (64\%) underwent RP only, while the remaining 58 (36\%) also underwent lymph node dissection. Positive lymph nodes were identified in only $4(7 \%)$ cases. $\mathrm{RP}$ was performed at a university hospital for 93 patients $(58 \%)$, while the remaining patients underwent RP at community centres.

Concomitant hormonal therapy was seldom prescribed before $2010(13 \%)$, but was much more frequent after 2010 $(37 \%)(p=0.003)$. Treatment with luteinizing hormonereleasing hormone agonists did not exceed 6 months in most cases (data not included). Post-RP patho-clinical features were comparable between both time periods: $\mathrm{pT}$-stage $(p=0.74)$, Gleason score $(p=0.10)$, and PSADT $(p=0.21)$ (Table 1).

\begin{tabular}{|c|c|c|c|}
\hline Factor & $\begin{array}{c}<2010 \\
(n=94)\end{array}$ & $\begin{array}{c}>2010 \\
(n=67)\end{array}$ & $p$ value \\
\hline $\begin{array}{l}\text { Mean age } \\
\text { (SD) }\end{array}$ & $64(6.4)$ & $63(7.0)$ & 0.6 \\
\hline $\begin{array}{l}\text { RT to pelvic } \\
\text { lymph nodes }\end{array}$ & $28 \%$ & $23 \%$ & 0.58 \\
\hline $\begin{array}{l}\text { Concomitant } \\
\text { ADT }\end{array}$ & $13 \%$ & $37 \%$ & 0.003 \\
\hline PSM & $65 \%$ & $70 \%$ & 0.5 \\
\hline pT stage & & & 0.74 \\
\hline pT2 & $45 \%$ & $43 \%$ & \\
\hline рT3a & $33 \%$ & $39 \%$ & \\
\hline pT3b & $22 \%$ & $18 \%$ & \\
\hline Gleason score & & & 0.10 \\
\hline 6 & $23 \%$ & $11 \%$ & \\
\hline 7 & $57 \%$ & $64 \%$ & \\
\hline $8-10$ & $19 \%$ & $26 \%$ & \\
\hline PSADT & & & 0.21 \\
\hline$<6$ months & $43 \%$ & $58 \%$ & \\
\hline 6-12 months & $31 \%$ & $19 \%$ & \\
\hline$>6$ months & $25 \%$ & $23 \%$ & \\
\hline \multicolumn{4}{|c|}{$\begin{array}{l}\text { SD: standard deviation; ADT: androgen deprivation therapy; PSM: positive surgical margin; } \\
\text { PSADT: prostate-specific antigen doubling time. Variables include mean age (years) with } \\
\text { standard deviation (SD) before RT, irradiation of pelvic lymph nodes, administration of } \\
\text { concomitant hormone therapy (ADT) and positive surgical margins (PSM). The pT3 group } \\
\text { was subdivided according to seminal vesicle invasion. PSA doubling time (PSADT) was } \\
\text { calculated between surgery and RT. }\end{array}$} \\
\hline
\end{tabular}

The median PSA value prior to RT consultation however decreased from $0.46 \mu \mathrm{g} / \mathrm{L}$ (before 2010) to $0.26 \mu \mathrm{g} / \mathrm{L}$ (after 2010) $(p=0.001)$ (Table 2).

Throughout the study period, only 6 patients were treated within 120 days after surgery, whereas 20 patients (12.4\%) were treated within 180 days after surgery. We calculated the time interval between surgery and first referral to radiation oncology. The median time was more than halved, dropping from 672 days before 2010 to 300 days after $2010(p=0.04)$. On the other hand, the time interval between first referral and treatment ( 7 weeks) remained unchanged $(p=0.82)$. When these results were compounded, the median time between surgery and first RT dropped from 727 days to

\begin{tabular}{lccc}
\hline \multicolumn{3}{l}{ Table 2. PSA before RT according to different cut-offs } \\
\hline PSA before RT & $\begin{array}{c}<\mathbf{2 0 1 0} \\
(\mathbf{n}=\mathbf{9 4 )}\end{array}$ & $\begin{array}{c}>\mathbf{2 0 1 0} \\
(\mathbf{n}=\mathbf{6 7})\end{array}$ & $\boldsymbol{p}$ value \\
\hline$>1.0 \mu \mathrm{g} / \mathrm{L}$ & $23 \%$ & $11 \%$ & 0.06 \\
$<0.5 \mu \mathrm{g} / \mathrm{L}$ & $50 \%$ & $75 \%$ & 0.002 \\
$<0.3 \mu \mathrm{g} / \mathrm{L}$ & $42 \%$ & $71 \%$ & 0.001 \\
$<0.2 \mu \mathrm{g} / \mathrm{L}$ & $18 \%$ & $28 \%$ & 0.18 \\
\multirow{2}{*}{ Median PSA ( $\mu \mathrm{gg} / \mathrm{L})(\mathrm{IOR})$} & 0.46 & 0.26 & 0.001 \\
\hline
\end{tabular}

PSA: prostate-specific antigen; RT: radiation therapy; IQR: interquartile range. 


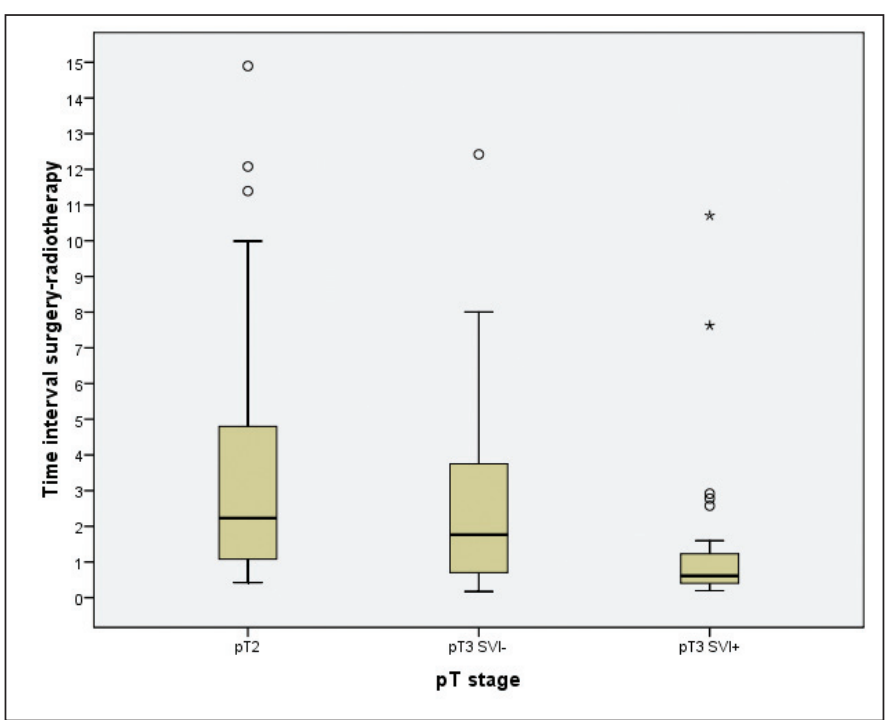

Fig. 1. Time interval between surgery and first radiation therapy (years) throughout the study period, according to pathological stage. SVI+: seminal vesicle invasion; SVI- : no seminal vesicle invasion. The results are expressed as boxplots. Rectangles depict interquartile range (IOR), and the middle line in the box, the median. Whiskers extend as far as the data, but not more than 1.5 times above the 75th percentile and below the 25th percentile. The "0" indicates values more than 1.5 IORs, but less than 3 IORs. Asterisks $\left({ }^{*}\right)$ indicate values above 3 IQRs.

404 days ( $p=0.03$ ) (Table 3). The time interval between surgery and first RT significantly correlated with pathological stage $(R=-0.264, p=0.001)$, Gleason score $(R=-0.223$, $p=0.005)$ and PSADT $(\mathrm{R}=0.529, p<0.001)$ (Fig. 1, Fig. 2, Fig. 3).

The mean follow-up after RT was 61 months for patients treated before 2010 and 29 months for those treated after

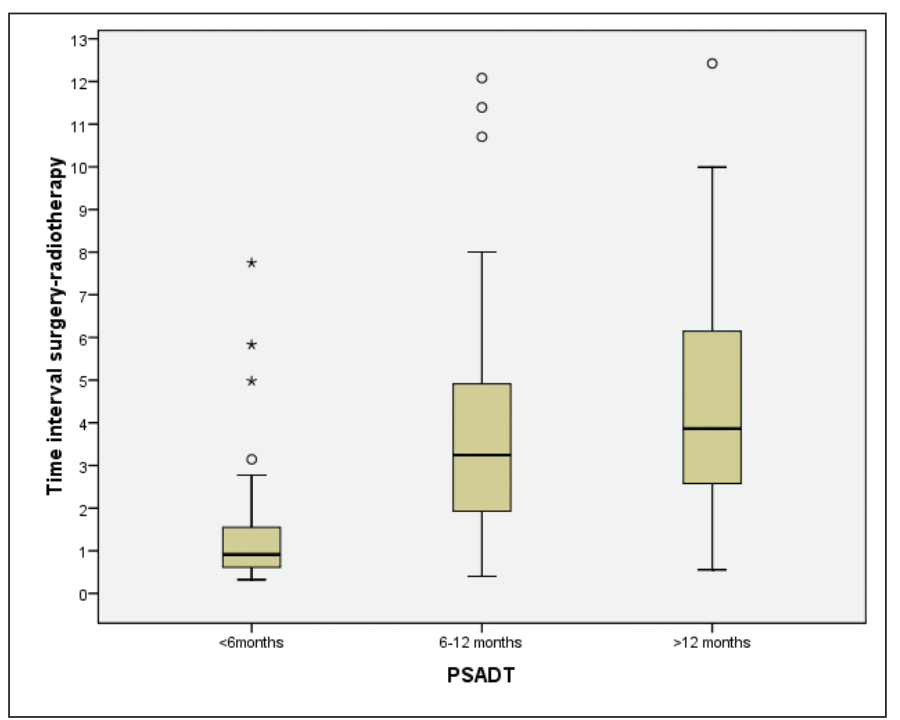

Fig. 3. Time interval between surgery and first radiation therapy (years) throughout the study period, according to prostate-specific antigen doubling time (PSADT).

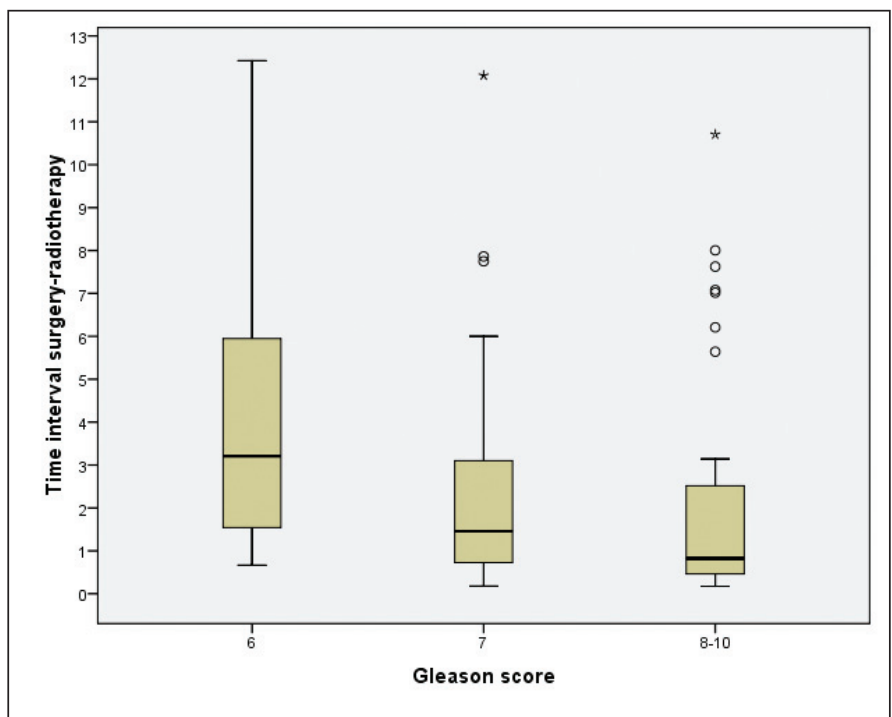

Fig. 2. Time interval between surgery and first radiation therapy (years) throughout the study period, according to Gleason score.

$2010(p<0.001)$. Androgen deprivation therapy (ADT) was started in $24 \%$ of patients treated before 2010 and $28 \%$ of patients treated after $2010(p=0.81)$. ADT was started 27 months after RT in patients treated before 2010 and 15 months for patients treated after $2010(p=0.04)$. Only 4 patients died of prostate cancer and 3 of unrelated cancers. Pelvic lymph node recurrence was documented in 6 patients and bone metastasis in 7 patients, including 4 patients displaying progression in both.

\section{Discussion}

We conducted a retrospective study to evaluate whether referral practices after RP changed significantly with publication of the SWOG 8794 Trial in late 2009. We report the single-practice experience of 161 patients treated over a decade (2004-2014). No study has recently evaluated the impact of any of the 3 major adjuvant studies on current practice..$^{1-3}$ The only relatively similar study we found was one based on the Surveillance, Epidemiology and End Results SEER Cancer Registry from 2000 to 2007. ${ }^{5}$ The latter authors identified 21927 patients who underwent $\mathrm{RP}$ for non-metastatic (NOMO) prostate cancer with adverse pathological features (pT3 or margin-positive pT2). The recommendation rate for post-prostatectomy radiation did not change over time, remaining constant at $13.5 \%$.

Our results demonstrate that the median time interval between RP and first referral for RT dropped significantly $(p=0.04$ ) from 672 days (before 2010) to 300 days (after 2010). It is therefore likely that referring urologists have translated SWOG 8794 trial results early into clinical practice, well ahead of international consensus guidelines. Moreover, it was not until 2013 that the American Society for Radiation 


\begin{tabular}{|c|c|c|c|}
\hline & $<2010(n=94)$ & $>2010(n=67)$ & $p$ value \\
\hline Time from surgery $\rightarrow$ first referral & $672(295-1449)$ & $300(225-1023)$ & 0.04 \\
\hline Time from first referral $\rightarrow$ first radiation therapy & $49(30-94)$ & $53(27-103)$ & 0.82 \\
\hline Time from surgery $\rightarrow$ first radiation therapy & $727(231-1260)$ & $404(132-995)$ & 0.03 \\
\hline
\end{tabular}

The results are expressed as median intervals in days (with corresponding interquartile range).

Therapy and the American Urological Association issued a joint recommendation. They recommended that physicians offer adjuvant RT to patients with adverse pathological features at RP (Standard; Evidence Strength Grade A) and salvage RT to patients with PSA or local recurrence after RP in whom there is no evidence of distant metastatic disease (Recommendation; Evidence Strength Grade C). ${ }^{6}$

Even though the median time interval between surgery and first RT was nearly halved after 2010, it still amounts to a bit more than 13 months (404 days), meaning that most patients are actually treated in salvage rather than pure adjuvant settings. A currently unresolved issue is whether adjuvant RT should be administered within 6 months after surgery for every patient with undetectable PSA and unfavourable risk factors versus delaying salvage RT until the postoperative PSA exceeds $0.2 \mu \mathrm{g} / \mathrm{L}$. In a large, matched, case-control study (890 patients) evaluating adjuvant and early salvage RT (given at PSA $\leq 0.5 \mu \mathrm{g} / \mathrm{L}$ ), no differences in 2- and 5-year biochemical recurrence-free survival were found, even when patients were stratified according to pT3 sub-stages and surgical margin status (all $p \geq 0.4$ ). ${ }^{7}$ Similar results were obtained when the cut-off to define early salvage RT was set at PSA $0.3 \mu \mathrm{g} / \mathrm{L}$ (all $p \geq 0.5$ ). The apparently equivalent efficacy of early salvage with adjuvant RT observed in this retrospective study still needs to be confirmed with prospective data.

We demonstrated a significant drop over time in median PSA values before RT $(p=0.001)$, from $0.46 \mu \mathrm{g} / \mathrm{L}$ (before 2010 ) to $0.26 \mu \mathrm{g} / \mathrm{L}$ (after 2010). This is also shown in that three-quarters of patients after 2010 were treated with a value $<0.5 \mu \mathrm{g} / \mathrm{L}$ compared to only $50 \%$ before 2010 . As lower PSA levels correlate with reduced tumour burden, better longterm outcomes might be expected among patients treated at lower PSA counts. It still remains debatable, in the setting of salvage RT after RP, if RT should always be started at the lowest PSA possible. It has been reported, for example, that a subset of patients with biochemical recurrence $(8.8 \%)$ after RP may have detectable but stable PSA levels for 10 years or more, with no evidence of clinical progression. ${ }^{8}$

Since 2010, RT after RP was initiated at our centre at PSA $<0.2 \mu \mathrm{g} / \mathrm{L}$ in nearly $30 \%$ of cases. It might now be tempting, with ultrasensitive assays, to define either lower cut-offs, or integrate Radiation Therapy Oncology Group (RTOG) criteria (2 consecutively-rising values) in the setting of PSA $<0.2 \mu \mathrm{g} / \mathrm{L}$, or even both, especially in patients with
PSM. This issue remains unresolved, and patients with very low PSA $(0.1-0.2 \mu \mathrm{g} / \mathrm{L})$ are currently included in the RTOG 0534 Trial. ${ }^{9}$

Two-thirds of patients in our study, who received RT after RP, had PSM, a known risk factor for biochemical recurrence. The impact of PSM on cancer-specific mortality is less clear. In a large, multi-institutional, retrospective study of 11521 men subjected to RP between 1987 and 2005, PSM were not significantly associated with excess cancerspecific mortality, after adjusting for important clinical and pathological parameters, including postoperative RT. ${ }^{10}$ Early RT (PSA $<0.5 \mu \mathrm{g} / \mathrm{L}$ ) was not linked in this study with increased cancer-specific mortality, probably because of $\mathrm{RT}^{\prime}$ 's protective effect. On the other hand, the risk of cancerspecific mortality was greater among men receiving late salvage RT (PSA $>0.5 \mu \mathrm{g} / \mathrm{L}$ ). These results were unlikely to be causative, but probably attributable to the fact that most men with post-prostatectomy PSA $>0.5 \mu \mathrm{g} / \mathrm{L}$ have distant micrometastatic disease and are thus unlikely to benefit from salvage RT.

We documented a sharp increase of ADT use over time. Since 2010, it has been prescribed to more than one-third of our patients. ADT would be endorsed by $35 \%$ of Australian and New Zealand radiation oncologists for high-risk prostate cancer patients after surgery, according to a pre-meeting practice survey in November 2012. ${ }^{11}$ It reflects a global trend in daily practice to recommend ADT addition off-study to treat patients with unfavourable features. ${ }^{12}$ To the best of our knowledge, ADT combined with RT in salvage settings post-RP is supported only by retrospective data, not by any strong prospective phase 3 study (unlike frontline, primary, combined treatment for non-operated prostate cancer). The definitive role of ADT in salvage settings is expected to be identified by the large, ongoing, prospective RTOG 0534 Trial.

The change in practice demonstrated in our study might be a direct consequence of the overall survival benefit demonstrated by SWOG 8794. It could also reflect better awareness of higher PSA levels as an adverse prognostic factor after the publication of the paper by Stephenson and colleagues ${ }^{13}$ as well as increased collaboration between urooncologists and ROs.

Finally, we demonstrated a strong correlation between adverse factors (pT-stage, Gleason score and PSADT) and shorter time lag between surgery and first RT, throughout 
Taussky et al.

the study period. These results provide evidence of rigorous consistency in the risk-adjusted workup and treatment of patients in our multidisciplinary setting over the past decade.

Given the retrospective nature of our study, the mean follow-up was twice as long for patients treated before 2010 than for those treated thereafter. Despite this time bias, patients treated after 2010 were as likely to receive salvage ADT as patients treated before 2010, with first treatment starting on average 12 months earlier. This might reflect a trend towards more aggressive cancer phenotypes for patients recently treated.

Our study's limitations include its retrospective nature and the small number of patients studied. The publication of SWOG 8794 might not be the only reason for a shift towards an earlier referral, but also the use of ultrasensitive PSA tests. More importantly, it would have been highly relevant to address the issue as to whether the observed shift towards shorter referral time intervals leads to better clinical outcomes. Unfortunately, the lack of long-term, mature data (especially from patients treated after 2010) prevents us from evaluating the impact of observed practice pattern shifts in biochemical recurrence-free survival.

In our study, all patients were treated by a single radiation oncologist. This situation has the major advantage of cancelling any inter-observational bias, providing greater treatment homogeneity. The obvious drawback is that our experience might not reflect the practice of other radiation oncologists in the same department, and might not be immediately generalizable to any other tertiary-referral hospital or community-based oncology centres.

\section{Conclusion}

Our study demonstrates a positive impact of SWOG 8794 in earlier referral patterns following RP for secondary radiotherapy at a single Canadian, tertiary care centre. This change in practice might be a direct consequence of the overall survival benefit demonstrated by SWOG 8794. Optimal timing of RT after surgery still remains an unsolved issue. Ongoing prospective trials, such as NCIC PR-13, might provide better insight into this longstanding debate. We therefore encourage all urologists and radiation oncologists to participate in this trial.
Competing interests: Dr. Alenizi and Dr. Taussky declare no competing financial or personal interests. Michel Zimmermann was funded by the CHUM Foundation and Paladin. Dr. Kevin Zorn is a paid consultant and proctor for Greenlight for American Medical Systems.

This paper has been peer-reviewed.

\section{References}

1. Bolla M, van Poppel H, Tombal B, et al. Postoperative radiotherapy after radical prostatectomy for high-risk prostate cancer: Long-term results of a randomised controlled trial (EORTC Trial 22911). Lancet 2012;380:2018-27. http://dx.doi.org/10.1016/S0140-6736(12)61253-7

2. Thompson IM, Tangen $C M$, Paradelo J, et al. Adjuvant radiotherapy for pathological T3NOMO prostate cancer significantly reduces risk of metastases and improves survival: Long-term followup of a randomized clinical trial. J Urol 2009;181:956-62. http://dx.doi.org/10.1016/i.juro.2008.11.032

3. Wiegel T, Bottke D, Steiner U, et al. Phase III postoperative adjuvant radiotherapy after radical prostatectomy compared with radical prostatectomy alone in pT3 prostate cancer with postoperative undetectable prostate-specific antigen: ARO 96-02/AUO AP 09/95. J Clin Oncol 2009;27:2924-30. http://dx.doi.org/10.1200/JC0.2008.18.9563

4. Showalter TN, Foley KA, Jutkowitz $E$, et al. Costs of early adjuvant radiation therapy after radical prostatectomy: A decision analysis. Ann Oncol 2012;23:701-6. http://dx.doi.org/10.1093/annonc/ mdr281

5. Hoffman KE, Nguyen PL, Chen MH, et al. Recommendations for post-prostatectomy radiation therapy in the United States before and after the presentation of randomized trials. J Urol 2011;185:116-20.

6. Valicenti RK, Thompson I Jr, Albertsen P, et al. Adjuvant and salvage radiation therapy after prostatectomy: American Society for Radiation Oncology/American Urological Association guidelines. Int J Radiat Oncol Biol Phys 2013;86:822-8. http://dx.doi.org/10.1016/i.i.jobp.2013.05.029

7. Briganti $A$, Wiegel $T$, Joniau $S$, et al. Early salvage radiation therapy does not compromise cancer control in patients with pT3NO prostate cancer after radical prostatectomy: Results of a match-controlled multiinstitutional analysis. Eur Urol 2012;62:472-87. http://dx.doi.org/10.1016/i.eururo.2012.04.056

8. Shinghal R, Yemoto C, McNeal JE, et al. Biochemical recurrence without PSA progression characterizes a subset of patients after radical prostatectomy. Urology 2003;61:380-5. http://dx.doi.org/10.1016/ S0090-4295(02)02254-98)

9. RTOG 0534 Protocol Information. Last accessed February 20, 2015. http://www.Itog.org/clinicaltrials/ protocoltable/studydetails.aspx?study=0534. Accessed August 18, 2015.

10. Stephenson A, Eggener SE, Hernandez AV, et al. Do margins matter? The influence of positive surgical margins on prostate cancer-specific mortality. Eur Urol 2014;65:675-80. http://dx.doi.org/10.1016/i. eururo.2013.08.036

11. Lehman M, Sidhom M, Kneebone AB, et al. FROGG high-risk prostate cancer workshop: Patterns of practice and literature review. Part II post-radical prostatectomy. J Med Imaging Radiat Oncol 2014;58:392-400. http://dx.doi.org/10.1111/1754-9485.12139

12. Roach $M$ 3rd. Current trends for the use of androgen deprivation therapy in conjunction with radiotherapy for patients with unfavorable intermediate-risk, high-risk, localized, and locally advanced prostate cancer. Cancer 2014;120:1620-9. hittp://dx.doi.org/10.1002/cncr.28594

13. Stephenson AJ, Scardino PT, Kattan MW, et al. Predicting the outcome of salvage radiation therapy for recurrent prostate cancer after radical prostatectomy. J Clin Oncol 2007;25:2035-41. http://dx.doi. org/10.1200/JC0.2006.08.9607

Correspondence: Dr. Daniel Taussky, Division of Urology, Centre Hospitalier de l'Université de Montréal, QC; daniel.taussky.chum@ssss.gouv.qc.ca 